\title{
A SELF-ORGANIZATION PHENOMENON IN THE LASER SYNTHESIS OF NITRIDES ON TITANIUM, ZIRCONIUM AND HAFNIUM SURFACES IN A LIQUID NITRIGEN MEDIUM
}

T.N. KHATKO

Physical and Engineering Institute of Academy of Sciences of BSSR, Zhodinskaya 4, Minsk 220730, USSR

It is shown theoretically and experimentally that a chemical medium subject to laser radiation acquires the ability to selforganization. This process can be controlled by changing laser beam parameters, i.e. intensity, a beam radius and wavelength. It is known that on titanium, zirconium and hafnium surfaces placed into liquid nitrogen and subjected to laser radiation there proceeds chemical synthesis of nitrides. Here a melting surface chemically interacting with nitrogen plays the main role.In the present work it has been revealed experimentally that at millisecond IR lager irradiation $(\lambda=1.6 \mu \mathrm{m})$ of titanium, zirconium and hafnium surfaces being in a liquid nitrogen medium there is observed a self-organization phenomenon in a thin surface layer of melted metal interacting with nitrogen which results in formation of a 5-20 um layer with a structure that differs essentially from that of a melting zone. Scanning electron microscopy and metallographic examination have revealed that the surface layer of a melting zone formed on a titanium surface consists of columnar TiN crystals having the same size and being equidistant from each other. Similar structures have been observed on zirconium and hafnium surfaces. A cross size and height of TiN crystals as well as a distance between them have depended on laser radiation power density : $q=10^{5}$ $\left.10^{8} \mathrm{~W} \cdot \mathrm{cm}^{-2}\right)$, impulse duration $(\tau=1-6 \mathrm{~m}: \mathrm{s})$ and a spot radius $(r=500-2000 \mu \mathrm{m})$. At $q=1,2 \cdot 10^{6} \mathrm{~W} \cdot \mathrm{cm}^{-2}, \tau=4 \mathrm{~ms}$ and $r=1 \mathrm{~mm}$ the above parameters have amounted to 1.5 ; 10 and $2.5 \mathrm{\mu m}$, respectively. 